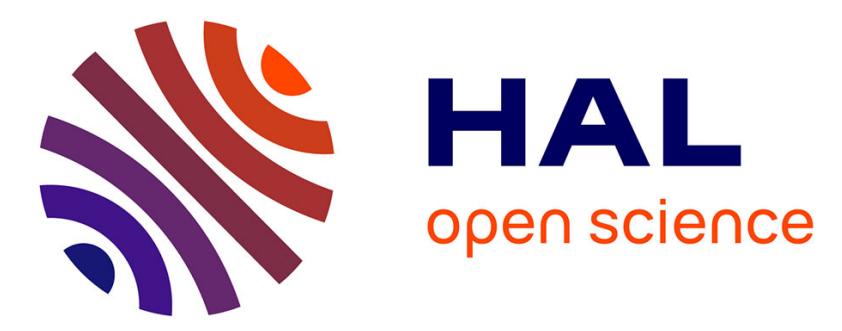

\title{
Kelvin Probe Force Microscopy and Electrostatic Force Microscopy responses to the polarization in a ferroelectric thin film: theoretical and experimental investigations \\ Mireille Cuniot-Ponsard
}

\section{To cite this version:}

Mireille Cuniot-Ponsard. Kelvin Probe Force Microscopy and Electrostatic Force Microscopy responses to the polarization in a ferroelectric thin film: theoretical and experimental investigations. Journal of Applied Physics, 2013, 114 (1), pp.014302. 10.1063/1.4812393 . hal-00841425

HAL Id: hal-00841425

https://hal-iogs.archives-ouvertes.fr/hal-00841425

Submitted on 18 Nov 2015

HAL is a multi-disciplinary open access archive for the deposit and dissemination of scientific research documents, whether they are published or not. The documents may come from teaching and research institutions in France or abroad, or from public or private research centers.
L'archive ouverte pluridisciplinaire HAL, est destinée au dépôt et à la diffusion de documents scientifiques de niveau recherche, publiés ou non, émanant des établissements d'enseignement et de recherche français ou étrangers, des laboratoires publics ou privés. 


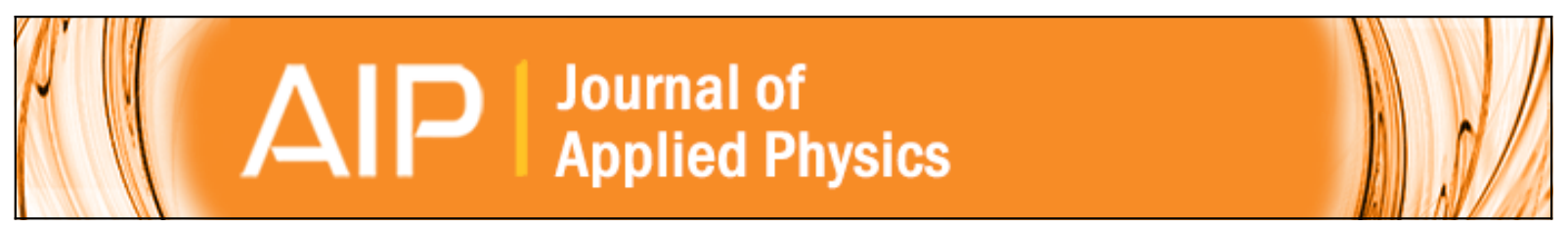

Kelvin probe force microscopy and electrostatic force microscopy responses to the polarization in a ferroelectric thin film: Theoretical and experimental investigations

M. Cuniot-Ponsard

Citation: Journal of Applied Physics 114, 014302 (2013); doi: 10.1063/1.4812393

View online: http://dx.doi.org/10.1063/1.4812393

View Table of Contents: http://scitation.aip.org/content/aip/journal/jap/114/1?ver=pdfcov

Published by the AIP Publishing

\section{Articles you may be interested in}

Effect of tip polarity on Kelvin probe force microscopy images of thin insulator CaF2 films on $\mathrm{Si}(111)$

Appl. Phys. Lett. 101, 083119 (2012); 10.1063/1.4748291

Kelvin probe force microscopy-based characterization techniques applied for electrostatic MEMS/NEMS devices and bare dielectric films to investigate the dielectric and substrate charging phenomena

J. Vac. Sci. Technol. A 29, 051101 (2011); 10.1116/1.3611004

Increase in carrier mobility of organic ultrathin-film transistor with increasing molecular layers investigated by Kelvin probe force microscopy

J. Appl. Phys. 97, 124503 (2005); 10.1063/1.1937474

Surface potential of ferroelectric thin films investigated by scanning probe microscopy

J. Vac. Sci. Technol. B 17, 1930 (1999); 10.1116/1.590851

Kelvin probe force microscopy

Appl. Phys. Lett. 58, 2921 (1991); 10.1063/1.105227

\section{AlP $\left.\right|_{\text {APL Photonics }}$}

APL Photonics is pleased to announce Benjamin Eggleton as its Editor-in-Chief

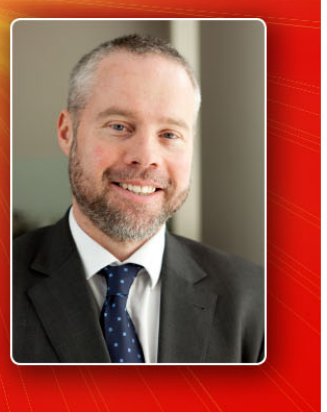




\title{
Kelvin probe force microscopy and electrostatic force microscopy responses to the polarization in a ferroelectric thin film: Theoretical and experimental investigations
}

\author{
M. Cuniot-Ponsard \\ Laboratoire Charles Fabry, IOGS, CNRS, Univ Paris-Sud, 2 avenue Augustin Fresnel, RD 128, \\ 91127 Palaiseau cedex, France
}

(Received 28 May 2013; accepted 11 June 2013; published online 2 July 2013)

\begin{abstract}
The ability of non-contact electric modes (Kelvin probe force microscopy (KPFM) and electrostatic force microscopy $(\mathrm{EFM})$ ) to provide quantitative information about the remnant polarization in a ferroelectric thin film is theoretically and experimentally investigated. The theoretical relationship between the microscopic signal and the polarization in the film is established: it is linear in the KPFM mode and parabolic in the EFM mode. The KPFM and EFM signals are predicted tip-shape, tip-surface distance, and poled area dimensions dependent so that an absolute value of the polarization cannot be extracted. Nevertheless provided these parameters are held constant, KPFM theoretically enables to investigate quantitatively the polarization dependence on any parameter relative to the film preparation conditions or to the poling process. It does not enable to investigate its dependence on poled area geometry. Based on these conclusions, experimental KPFM investigations have been carried out on a ferroelectric strontium barium niobate $\left(\mathrm{Sr}_{\mathrm{x}} \mathrm{Ba}_{1-\mathrm{x}} \mathrm{Nb}_{2} \mathrm{O}_{6}, \mathrm{SBN}: 100 \mathrm{x}\right)$ epitaxial thin film deposited on a platinum covered $\mathrm{MgO}$ substrate. Poled areas are created in the film by moving the biased tip in contact with the surface. KPFM images are dominated by the written polarization and not by injected or attracted extrinsic charges. The experimental decay of the KPFM signal with increasing tip-surface distance confirms the contribution of the volume of the lever/tip to the KPFM response. The spontaneous evolution of the written polarization as well as the polarization dependence on poling voltage and poling duration are investigated using KPFM, with the poled area dimensions as a parameter. For comparison, the macroscopic remnant polarization in the SBN film has been measured versus poling field from hysteresis cycles drawn using a macroscopic Pt dot as top contact. The significant difference observed between macroscopic and microscopic results can be accounted for by using a simple model of the inhomogeneous electric field generated by a poling tip. KPFM measurements do not alter the initial polarization of the film and can provide essential qualitative and quantitative information on the polarization in a ferroelectric thin film. (C) 2013 AIP Publishing LLC.

[http://dx.doi.org/10.1063/1.4812393]
\end{abstract}

\section{INTRODUCTION}

Kelvin Probe Force Microscopy (KPFM), ${ }^{1,2}$ also referred to as Scanning Surface Potential Microscopy (SSPM), is a nanometer-scale adaptation of the macroscopic Kelvin-Zisman probe method ${ }^{3,4}$ used to measure the contact potential difference (CPD) between two materials. Despite a simple principle, the KPFM measurements have a reputation for being difficult. Even in the case of perfectly conductive tip and sample, Colchero et al. ${ }^{5}$ demonstrated that the value theoretically obtained with the usual KPFM operating mode in air (force detection, amplitude modulation) is not the true tip-sample local CPD, but a complex weighted average, strongly tip shape and tip-surface distance dependent. Moreover, contamination of the surface and screening effects contribute to blur the intrinsic surface potential contrast. Only KPFM measurements performed using force gradient detection (frequency modulation $(\mathrm{FM}))^{5,6}$ under ultra-high vacuum conditions should be able to provide a reliable value of the local tip-sample CPD. Additionally, KPFM measurements on semiconductors may be affected by the existence of the voltage-dependent space charge capacity of the semiconductor, then leading to erroneous conclusions. ${ }^{7,8}$ Finally, the KPFM standard theory, where the tip-sample system is modeled as a capacitor, does not hold true in the case of insulating samples. The KPFM image of an insulating surface is not a CPD map. Its interpretation requires a model of the tip-sample interaction which strongly varies with the sample configuration and features. ${ }^{9-14}$

The use of KPFM to map qualitatively the relative strength and direction of the dielectric polarization in ferroelectric materials has been continuously reported for 13 years. ${ }^{10,15-22}$ In these reports, KPFM images often appear governed by the presence of extrinsic charges due to the poling and/or screening processes. For this reason, the piezoresponse force microscopy (PFM), ${ }^{23}$ that is a contact mode much less sensitive to extrinsic effects, has remained by far the mainstream technique for characterization of the ferroelectric materials at the nanometer scale. A disadvantage of a contact mode like PFM is that the voltage between tip and bottom electrode is applied to the ferroelectric material instead of being mainly applied to the air gap in a 
non-contact mode. The initial ferroelectric polarization of the sample is therefore modified by the alternating voltage used to produce the piezoelectric movement of the surface. This modification must be held relatively negligible, which implies that the PFM technique is not suited for ferroelectric materials with a low coercive field. Moreover, a quantitative interpretation of PFM images is still less straightforward ${ }^{24,25}$ than that of KPFM images.

The drive towards miniaturization and the wide application potential of ferroelectric thin films has generated a great specific demand for a reliable characterization technique for polarization in these films at the nanometer scale. The present paper investigates the ability of non-contact electric modes (KPFM and EFM, ${ }^{26,27}$ acronym for Electrostatic Force Microscopy) to satisfy such a demand. Section II proposes a theoretical analysis of the contrast formation mechanism in the KPFM and EFM images of a polarized area in a ferroelectric thin film. The KPFM and EFM responses are first expressed in the most general case where they cannot be merely related to a contact potential difference, then they are calculated in the particular case of a polarized thin area. Experimental KPFM investigations based on the conclusions of this analysis have been carried out on ferroelectric strontium barium niobate $\left(\mathrm{Sr}_{\mathrm{x}} \mathrm{Ba}_{1-\mathrm{x}} \mathrm{Nb}_{2} \mathrm{O}_{6}, \mathrm{SBN}\right.$ :100x) epitaxial thin films, with the aim of extracting quantitative information about the local polarization dependence on poling parameters and about its spontaneous evolution with time. The details and results of these experiments are reported in Sec. III.

\section{THEORETICAL DEVELOPMENT}

\section{A. KPFM modeling}

In the conventional macroscopic Kelvin probe technique, ${ }^{3,4}$ a conductive vibrating probe is connected to a conductive or semi-conductive sample, the consequent alignment of Fermi levels generates a CPD between the vibrating probe and the sample surface, thus producing an alternating current in the probe-sample capacitor. This current is detected, and then nullified by adjusting the magnitude of a compensating DC voltage $\left(\mathrm{V}_{\mathrm{DC}}\right)$ applied between tip and bottom electrode. The Kelvin probe technique has been widely used to map the relative work function of metallic samples or the surface potential of semiconductors. In the KPFM adaptation, the above principle has been partially modified: (a) an additional $\mathrm{AC}$ voltage $\left(\mathrm{V}_{\mathrm{AC}} \cos \omega \mathrm{t}\right)$ is applied between tip and sample instead of mechanically oscillating the tip, and (b) the resulting harmonic oscillation of the tip is detected instead of a current in the tip-sample capacitor. Figure 1 schematically depicts the KPFM operation mode. The expression of the vertical electric force $d F_{z}$ acting on the surface element $d S$ of the conductive lever/tip is

$$
\begin{aligned}
d F_{z} & =-\frac{\sigma^{2}}{2 \varepsilon_{0}} \cos \theta d S \\
& =-\frac{-\left(\sigma_{S}+\sigma_{D C}+\sigma_{A C} \cos (\omega t-\varphi)\right)^{2}}{2 \varepsilon_{0}} \cos \theta d S,
\end{aligned}
$$

where $\sigma$ is the $d S$ surface charge density, $\sigma_{S}, \sigma_{D C}, \sigma_{A C}$ are the $d S$ surface charge densities induced, respectively, and

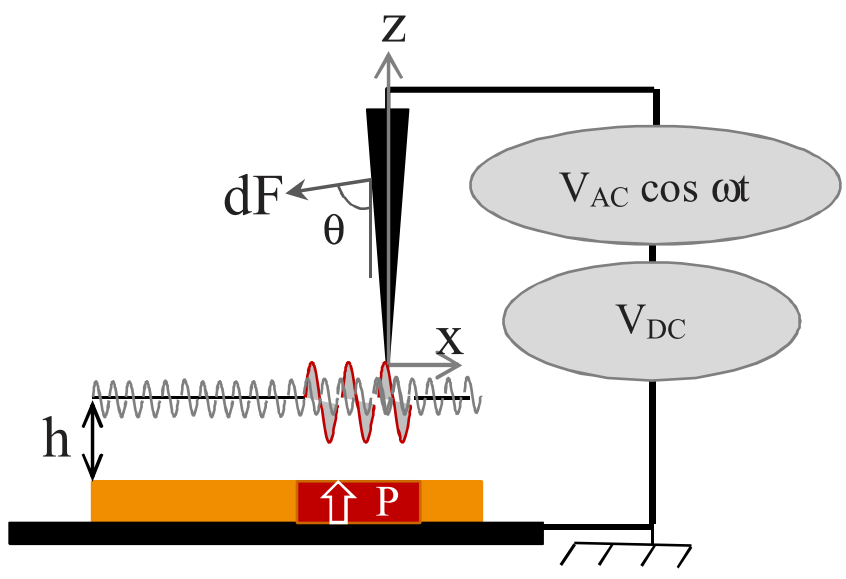

FIG. 1. Schematic representation of the KPFM operation mode. An AC voltage applied between a conductive tip and a sample induces a 3 -spectralcomponents force on the tip $(0, \omega, 2 \omega)$. The harmonic component is sensitive to the electric field generated by the sample (here a poled area in a ferroelectric film) and can be exactly nullified by applying a compensating $\mathrm{V}_{\mathrm{DC}}$ voltage (it is not nullified in the figure). A KPFM image is the map of this nulling $\mathrm{V}_{\mathrm{DC}}$ value when the tip scans the surface at a held constant mean distance $\mathrm{h}$.

separately, by the charge distribution in the sample, the DC voltage, and the $\mathrm{AC}$ voltage, $\varphi$ is the phase delay of the $\mathrm{AC}$ response, $\theta$ is the angle between the vector normal to the surface element $d S$ and the down vertical vector, and $\varepsilon_{0}$ is the vacuum dielectric permittivity. The surface charge densities $\sigma_{D C}$ and $\sigma_{A C}$ read

$$
\begin{aligned}
& \sigma_{D C}(x, y, z)=\frac{d C}{d S}(x, y, z) V_{D C}, \\
& \sigma_{A C}(x, y, z)=\frac{d C}{d S}(x, y, z) V_{A C},
\end{aligned}
$$

where $\mathrm{C}$ is the capacitance of the lever/tip-sample capacitor, and $(x, y, z)$ are the coordinates of any point on the lever/tip surface. The force $F_{z}$ on the lever/tip is therefore made of three spectral components

$$
\begin{gathered}
F_{z}(t)=F_{z, 0}+F_{z, \omega}(t)+F_{z, 2 \omega}(t) \\
F_{z, 0}=-\frac{1}{2 \varepsilon_{0}} \iint\left[\left(\sigma_{S}+\sigma_{D C}\right)^{2}+\frac{\sigma_{A C}^{2}}{2}\right] \cos \theta d S \\
F_{z, \omega}(t)=-\frac{1}{\varepsilon_{0}}\left[\iint\left(\sigma_{S}+\sigma_{D C}\right) \sigma_{A C} \cos \theta d S\right] \cos (\omega t-\varphi), \\
F_{z, 2 \omega}(t)=-\frac{1}{4 \varepsilon_{0}}\left[\iint \sigma_{A C}^{2} \cos \theta d S\right] \cos 2(\omega t-\varphi)
\end{gathered}
$$

The first component $F_{z, 0}$, static and attractive, acts on the mean tip-sample separation, the component $F_{z, \omega}$ produces an harmonic vertical oscillation of the tip, sensitive to the charge distribution on and in the sample, and the component $F_{z, 2 \omega}$ produces a vertical second harmonic oscillation of the tip, insensitive to a change in the electric properties of the sample or in the applied DC voltage (see Fig. 1). In the KPFM operation, the harmonic oscillation of the tip is 
synchronously detected through the correlated deflection of the cantilever, its amplitude is then nullified using a feedback loop that adjusts the magnitude of the DC voltage to a value $V_{D C}=V_{K}$ (referred to as "Kelvin voltage" in the following), so that the force $F_{z, \omega}(t)$ is maintained zero, otherwise expressed as

$$
\iint\left(\sigma_{S}+\sigma_{K}\right) \sigma_{A C} \cos \theta d S=0
$$

In the particular case of an homogeneous metallic sample,

$$
\sigma_{S}(x, y, z)=\frac{d C}{d S}(x, y, z) V_{C P D}
$$

where $V_{C P D}$ is the tip-sample contact potential difference, and Eq. (8) then may be written as

$$
\left(V_{C P D}+V_{K}\right) V_{A C}\left[\iint\left(\frac{d C}{d S}\right)^{2} \cos \theta d S\right]=0 .
$$

The harmonic force on the tip is then well nullified when the DC voltage and the CPD compensate each other. The difficulty pointed out by Colchero et $a l^{5}$ arises as soon as the $V_{C P D}$ value varies in the vicinity of the tip apex. These $V_{C P D}$ variations induce variations in the charge distribution $\sigma_{S}(x, y, z)$ on the lever/tip surface, and Eq. (9) holds only if $V_{C P D}$ is replaced by a function $V_{C P D}(x, y, z)$ of the coordinates of the lever/tip surface element dS. The Kelvin voltage is then a complex weighted average of the contact potential difference in the vicinity of the tip apex, lever/tip shape, and tip-surface distance dependent

$$
V_{K}=-\frac{\iint V_{C P D}\left(\frac{d C}{d S}\right)^{2} \cos \theta d S}{\iint\left(\frac{d C}{d S}\right)^{2} \cos \theta d S} .
$$

The FM operation mode, ${ }^{5,6}$ that detects the force gradient instead of the force exerted on the tip, strongly enhances the relative contribution of the tip apex to the detected and nullified signal, and therefore partially solves the above difficulty.

When the sample is made of insulating parts, electrostatic charges may be present on but also below the sample surface. The induced surface charge density $\sigma_{S}$ on the lever/ tip then results from a charge/potential volume distribution in the sample and cannot be merely related to the local potential on the surface straight below the tip apex like it is in Eq. (9). The general expression of the Kelvin voltage is

$$
V_{K}=-\frac{\iint \sigma_{S} \frac{d C}{d S} \cos \theta d S}{\iint\left(\frac{d C}{d S}\right)^{2} \cos \theta d S} .
$$

This relation demonstrates that a virtually calculable $V_{K}$ value always exists that exactly nullifies the harmonic force on the lever/tip. And yet $\sigma_{S}$ and $\sigma_{K}$ do not compensate each other at each point of the lever/tip surface, except in the particular case of a metallic homogeneous sample. Since $\sigma_{K}+\sigma_{S} \neq 0$ in general, applying the Kelvin voltage nullifies the harmonic force but does not nullify the electric field in the space between tip and sample, and therefore does not nullify the difference between the tip and the sample surface electric potentials. Relation (12) also implies that $V_{K}$ is tipshape and tip-sample distance dependent, except again for a metallic homogeneous sample.

\section{B. EFM modeling}

In the EFM mode, a conductive lever/tip is mechanically oscillated near its resonance frequency $f_{0}$ at a held constant height $h$ above the surface. Any variation in the force acting on the tip shifts this resonance frequency and therefore modifies the amplitude A and the phase $\varphi$ of the tip oscillation. By synchronously detecting $\mathrm{A}$ and $\varphi$, while raster scanning the tip at a fixed tip-sample mean distance, EFM can thus provide two complementary images of the electric field above a surface. Figure 2 schematically depicts the EFM operation mode. Referring to Eq. (1), the vertical electrostatic force on the tip can be written as

$$
F_{z}=-\frac{1}{2 \varepsilon_{0}} \iint \sigma^{2} \cos \theta d S
$$

This force shifts the initial resonance frequency $f_{0}$ (no electric field) to a lower value $f_{0}^{\prime}$, according to the following approximate relationship: ${ }^{28}$

$$
f_{0}^{\prime} \approx f_{0}-\frac{f_{0}}{2 k}\left(\frac{d F_{z}}{d Z_{O}}\right)_{h},
$$

where $\mathrm{k}$ is the lever/tip spring constant, $Z_{0}$ and $\mathrm{h}$ are, respectively, the position and the mean position of the tip apex above the surface (see Fig. 2). The amplitude of the lever/tip oscillation is then modified by $\Delta A$

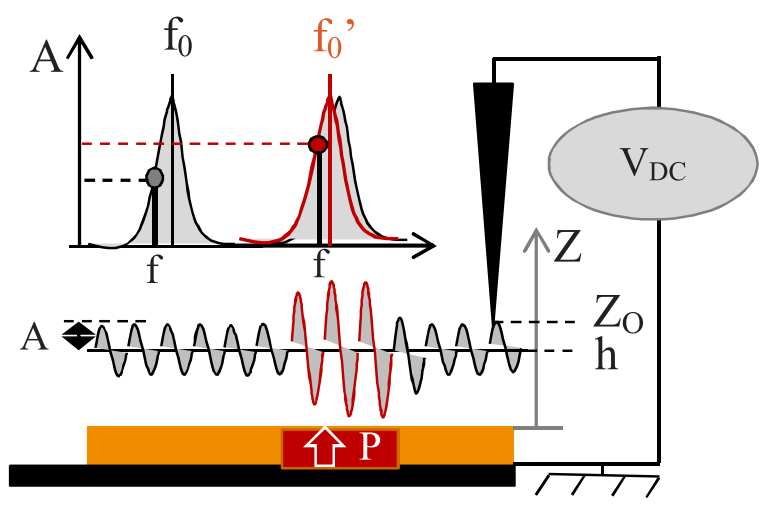

FIG. 2. Schematic representation of the EFM operation mode. A conductive tip is mechanically oscillated near its resonance frequency $f_{0}$ at a held constant height $h$ above the surface. An electric field generated by the sample (here a poled area in a ferroelectric film) modifies the force acting on the tip, shifts the resonance frequency $\left(f_{0} \rightarrow f_{0}^{\prime}\right)$, and increases the amplitude $\mathrm{A}$ of the tip oscillation. An EFM image is made of two maps: the tip oscillation amplitude and phase. A $V_{\text {DC }}$ voltage may be applied which allows up and down polarizations to be distinguished. 


$$
\begin{gathered}
\Delta A=\frac{d A}{d f_{0}} \Delta f_{0} \approx \frac{d A}{d f_{0}} \frac{f_{0}}{4 k \varepsilon_{0}} \frac{d\left[\iint \sigma^{2} \cos \theta d S\right]}{d Z_{O}}, \\
\Delta A \approx \frac{d A}{d f_{0}} \frac{f_{0}}{2 k \varepsilon_{0}} \iint \sigma\left(\frac{d \sigma}{d Z_{O}}\right)_{h} \cos \theta d S .
\end{gathered}
$$

The surface charge density on the lever/tip is generated by the charge distribution in the sample and, eventually, by a DC voltage applied between tip and sample: $\sigma=\sigma_{S}+\sigma_{D C}$. Taking Eq. (2) into account, the variation $\Delta A$ induced by the electric field above the surface can be expressed as

$$
\left.\left.\Delta A \approx \frac{d A}{d f_{0}} \frac{f_{0}}{2 k \varepsilon_{0}}\left[\iint \sigma_{S}\left(\frac{d \sigma_{S}}{d Z_{O}}\right)_{h} \cos \theta d S+V_{D C}\left[\iint\left(\frac{d \sigma_{S}}{d Z_{O}}\right)_{h} \frac{d C}{d S} \cos \theta d S+\iint \sigma_{S}\left(\frac{d\left(\frac{d C}{d S}\right)}{d Z_{O}}\right)\right)_{h} \cos \theta d S\right]+V_{D C}^{2} \iint_{d S} \frac{d\left(\frac{d C}{d S}\right)}{d Z_{O}}\right)_{h}^{\cos \theta d S}\right] .
$$

The first term of this expression is insensitive to the sign of $\sigma_{S}$. If $V_{D C}=0$, the contrast in the EFM image arises only from variations in the absolute value of the electric field above the surface. If $V_{D C} \neq 0$, the second term of Eq. (17) adds to $\triangle A$ a contribution sensitive to the sign of $\sigma_{S}$ and proportional to $V_{D C}$, which enables the EFM image to reveal any variation in the electric field above the surface. Nevertheless the first and second terms may blur each other and yield a confused contrast. The third term in Eq. (17) is insensitive to the charge distribution in the sample. The resulting variation in amplitude $\Delta A$ is therefore a complex function of the surface charge density induced on the lever/ tip by the sample. The variation $\Delta \varphi$ in the phase of the tip oscillation is obtained by replacing $A$ with $\varphi$ in Eq. (17). In the simple case of a metallic homogeneous sample, taking Eq. (9) into account, Eq. (17) reads

$$
\Delta A \approx \frac{d A}{d f_{0}} \frac{f_{0}}{2 k \varepsilon_{0}}\left(V_{C P D}+V_{D C}\right)^{2} \iint \frac{d C}{d S}\left(\frac{d\left(\frac{d C}{d S}\right)}{d Z_{O}}\right)_{h} \cos \theta d S
$$

Consistently, Eq. (18) predicts $\Delta A=0$ when the DC voltage and the contact potential difference compensate each other, that is when the electric field above the sample and therefore the induced charge on the lever/tip are nullified. As already discussed in the previous section, Eq. (18) does not hold true as soon as the CPD varies in the vicinity of the tip apex.

The comparison between Eqs. (12) and (17) provides evidence for a relationship between image and surface charge density on the lever/tip much more straightforward in the KPFM mode. Moreover, a disadvantage of the EFM mode is that the derivative $\frac{d A}{d f_{0}}$ cannot be considered as a constant when variations in the electric field, and consequently in the lever resonance frequency, are large.

\section{KPFM and EFM responses to the remnant polarization in a ferroelectric thin film}

We consider a ferroelectric thin film deposited on a grounded bottom electrode and calculate the electric field generated above the surface by a uniformly polarized area in this film. The area is chosen in the form of a disc and the field is calculated on the disc axis (see Fig. 3(a)). The remnant polarization $\vec{P}$ is represented perpendicular to the surface of the film in the figures but the following calculation holds for any $\vec{P}$ orientation. The disc generates an electric field identical to the field that would be generated by its top and bottom surfaces, respectively, charged with surface charge densities $+P_{Z}$ and $-P_{Z}$, where $P_{Z}$ is the polarization component perpendicular to the surface. This field induces a surface charge density $\sigma_{\mathrm{m}}$ in the underlying electrode. Finally, the field above the surface results from three contributions: the fields generated by the surface charge densities $+P_{Z},-P_{Z}$, and $\sigma_{\mathrm{m}}$, schematically represented in Fig. 3(b).
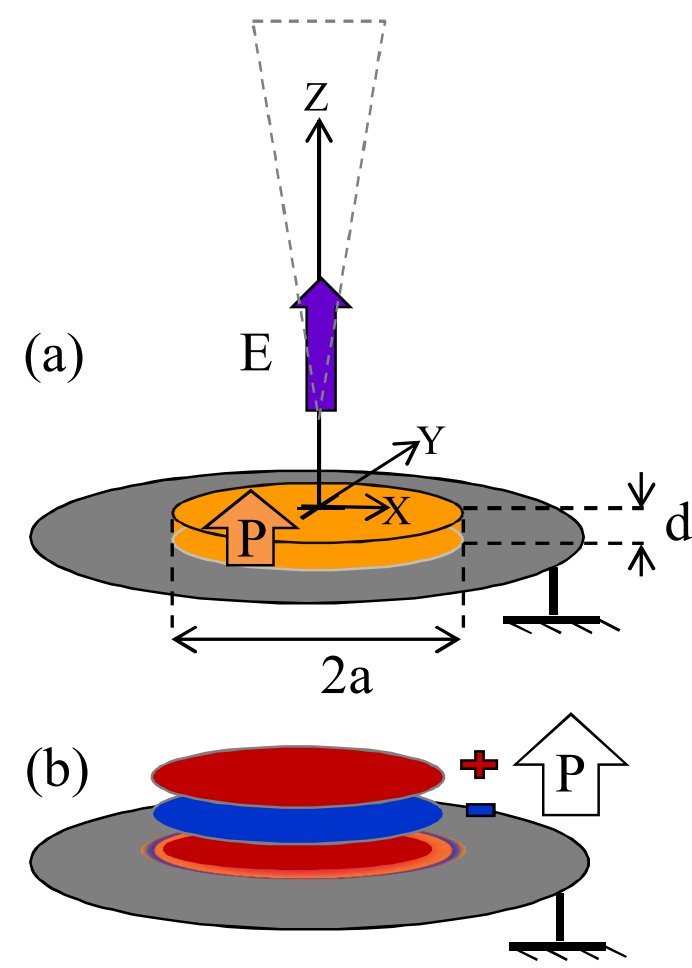

FIG. 3. Calculation of the electric field generated by a uniformly poled disc deposited on a grounded electrode at a point on its axis. (a) The poled disc generates an electric field identical to the field that would be generated by its top and bottom surfaces, respectively, charged with $+\mathrm{P}$ and $-\mathrm{P}$ surface charge densities. (b) This field induces a surface charge density $\sigma_{\mathrm{m}}$ in the underlying electrode. The field above the surface finally results from three contributions: the fields generated by the surface charge densities $+\mathrm{P},-\mathrm{P}$, and $\sigma_{\mathrm{m}}$. 
A classic calculation of the electric field $E$ created by a disc uniformly charged with a surface charge density $\sigma$, at a distance $\mathrm{Z}$ on the disc axis, yields

$$
E=\frac{\sigma}{2 \epsilon_{0}}\left(1-\frac{Z}{\sqrt{Z^{2}+a^{2}}}\right),
$$

where a is the radius of the disc. The electric field in the metallic electrode is necessarily zero which can therefore be expressed as

$$
\begin{gathered}
\frac{P_{Z}}{2 \epsilon_{0}}\left(1-\frac{d}{\sqrt{d^{2}+a^{2}}}\right)-\frac{P_{Z}}{2 \epsilon_{0}}+\frac{\sigma_{m}}{2 \epsilon_{0}}=0, \\
\sigma_{m}=P_{Z} \frac{d}{\sqrt{d^{2}+a^{2}}},
\end{gathered}
$$

where $\mathrm{d}$ is the thickness of the ferroelectric thin film. Taking into account Eqs. (19) and (21), the electric field on the $\mathrm{Z}$ disc axis above the surface can therefore be written as

$$
\begin{gathered}
E(0,0, Z)=\frac{1}{2 \epsilon_{0}}\left[P_{Z}\left(1-\frac{Z}{\sqrt{Z^{2}+a^{2}}}\right)-P_{Z}\left(1-\frac{Z+d}{\sqrt{(Z+d)^{2}+a^{2}}}\right)+P_{Z} \frac{d}{\sqrt{d^{2}+a^{2}}}\left(1-\frac{Z+d}{\sqrt{(Z+d)^{2}+a^{2}}}\right)\right], \\
E(0,0, Z)=\frac{P_{Z}}{2 \epsilon_{0}}\left[\frac{\alpha+\beta}{\sqrt{(\alpha+\beta)^{2}+1}}\left(1-\frac{\beta}{\sqrt{\beta^{2}+1}}\right)-\frac{\alpha}{\sqrt{\alpha^{2}+1}}+\frac{\beta}{\sqrt{\beta^{2}+1}}\right],
\end{gathered}
$$

where $\alpha=Z / a$ and $\beta=d / a$ are dimensionless. The function $2 \epsilon_{0} E / P_{Z}(\alpha, \beta)$ is represented in Figure 4. This figure brings into relief the fact that the electric field above a polarized surface will be hardly detectable if $d \ll a$ and/or $Z \gg a$. A polarized area in a ferroelectric thin film can therefore be easily detected only if its size is: (a) not too much larger than the film thickness $(a<20 d)$, (b) not too much small $(a>Z)$. KPFM and EFM usually scan the tip at a few tens of nanometers from the surface, which sets a first limit to the resolution that can be reached with these techniques: a polarized area will be hardly detected if its radius is lower than a few tens of nanometers.

Since $E(0,0, Z) \rightarrow_{a \rightarrow \infty} 0$, a non-polarized small area within a large polarized area generates a local electric field equal to that generated by the same small area polarized with the opposite polarization within a large non-polarized area.

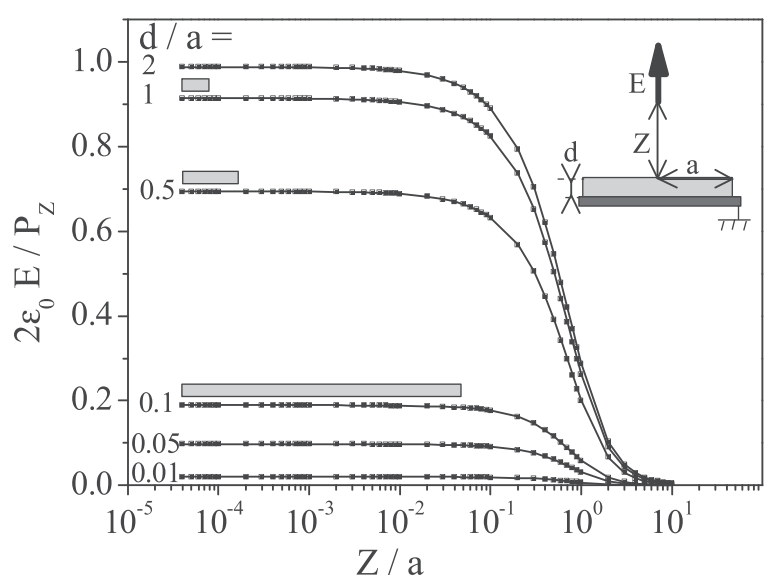

FIG. 4. Electric field E generated on its axis by a uniformly poled disc deposited on a grounded electrode, as a function of the distance $\mathrm{Z}$ from the surface. $P_{z}$ is the vertical component of the polarization, a is the radius of the disc, and $\mathrm{d}$ is its thickness. $\mathrm{E} / \mathrm{P}_{\mathrm{z}}$ is a function of the two dimensionless variables $\mathrm{Z} / \mathrm{a}$ and $\mathrm{d} / \mathrm{a}$. The electric field above a polarized surface will be hardly detectable if $d \ll a$ and/or $Z \gg a$.
The expression (23) is therefore valid whatever the polarization state of the film is around the disc provided $P_{Z}$ is replaced by the variation $\Delta P_{Z}=P_{Z}$ (disc) $-P_{Z}$ (film around).

It is also to be noted from Figure 4 that the electric field generated above a micrometer thick film by a given polarization is about three orders of magnitude lower than that generated by the same polarization in a crystal a few millimeters thick. The screening effect caused by extrinsic charges attracted on the ferroelectric surface is consequently expected significantly lower in the case of a ferroelectric thin film.

Figure 5 depicts the decrease of the electric field magnitude along a $\mathrm{Z}$ distance that corresponds to the usual height of a microscope tip $(\approx 12 \mu \mathrm{m})$ in the particular case of a film $0.6 \mu \mathrm{m}$ thick. It shows that the electric field generated in the volume of the tip may be strongly inhomogeneous or quasi uniform depending on the size of the polarized area. The surface charge density $\sigma_{S}(x, y, z)$ on the tip/lever surface that maintains a zero field in the volume of the conductive tip is dependent on the particular shape of the tip/lever, but is therefore also strongly dependent on the size and shape of the polarized area. This last point dooms to failure any attempt at quantifying $\sigma_{S}(x, y, z)$ and, further, the Kelvin voltage $\mathrm{V}_{\mathrm{KP}}$ induced by a polarization $P$. Nevertheless $\sigma_{S}(x, y, z)$ is proportional to $P_{Z}$ and Eq. (12) may be written as

$$
V_{K P}=-P_{Z} \frac{\iint f \frac{d C}{d S} \cos \theta d S}{\iint\left(\frac{d C}{d S}\right)^{2} \cos \theta d S}=-K P_{Z},
$$

where $\mathrm{f}$ is a function of $Z_{0} / a\left(Z_{0}\right.$ : position of the tip apex on the $\mathrm{Z}$ axis), $d / a$, the tip/lever particular shape, and the coordinates $(x, y, z)$ of the surface element $d S$.

The Kelvin voltage on the disc axis is therefore proportional to $P_{Z}$, while the relationship between the EFM signals and $P_{Z}$ resulting from Eq. (17) is in the form $\mathrm{A}_{1} P_{Z}^{2}$ $+\mathrm{A}_{2} V_{D C} P_{Z}+\mathrm{A}_{3} V_{D C}^{2}$. The coefficient $K$ in Eq. (24) is 


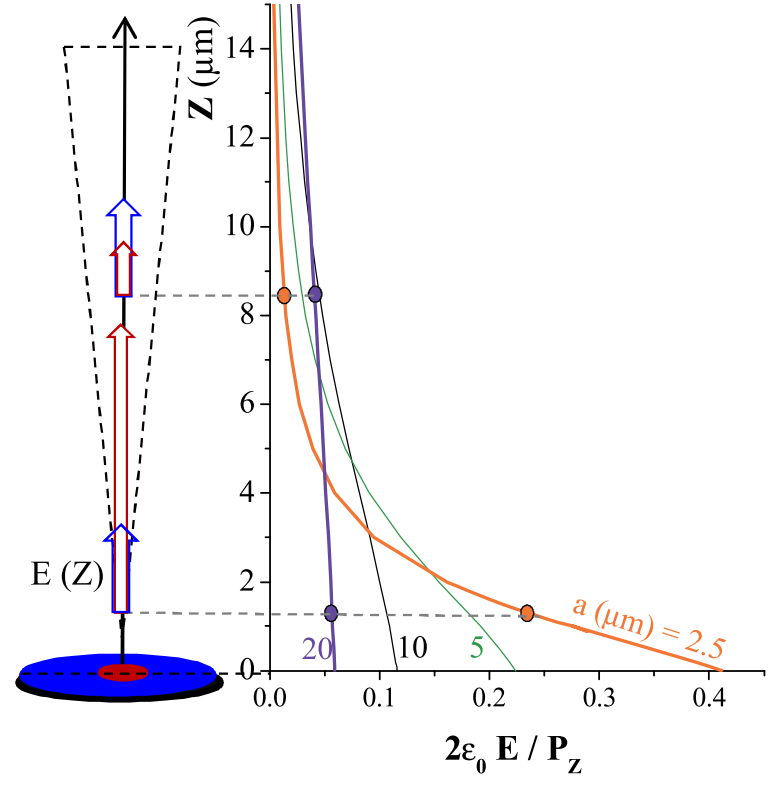

FIG. 5. Electric field magnitude along the height of a microscope tip set straight above the center of a poled disc. The particular case of a film $0.6 \mu \mathrm{m}$ thick has been chosen and the radius of the poled disc is a parameter. The electric field generated in the volume of the tip may be strongly inhomogeneous (red, $\mathrm{a}=2.5 \mu \mathrm{m}$ ) or quasi uniform (blue, $\mathrm{a}=20 \mu \mathrm{m}$ ) depending on the size of the polarized area.

hardly calculable even when regarding the simplest academic model made of a perfect conductive cone set on the axis of a uniformly polarized disc. It is not calculable as soon as a real lever/tip is used. However, relative measurements of $P_{Z}$ can be performed provided this coefficient $K$ is kept constant, which implies that the tip-surface distance $Z_{0}$, the thickness $d$, and radius $a$ of the polarized area are kept constant. The polarization dependence on any parameter relative to the film preparation conditions or to the poling process can therefore be quantitatively investigated using KPFM. On the other hand, KPFM cannot afford quantitative information about the polarization dependence on poled area geometry since the factor $\mathrm{K}$ is dependent on this geometry.

\section{EXPERIMENTAL INVESTIGATIONS}

\section{A. SBN ferroelectric thin films}

Strontium barium niobate $\left(\mathrm{Sr}_{\mathrm{x}} \mathrm{Ba}_{1-\mathrm{x}} \mathrm{Nb}_{2} \mathrm{O}_{6}\right.$, noted $\mathrm{SBN}: 100 \mathrm{x})$ is a ferroelectric material that crystallizes in the region $0.25<\mathrm{x}<0.75$ with the tetragonal tungsten bronze structure. ${ }^{29}$ SBN crystals exhibit an exceptionally high linear electro-optic coefficient, ${ }^{30}$ greatly exceeding that of the primary electro-optic material $\mathrm{LiNbO}_{3}$. The drive towards miniaturization and the development of new photonic components have oriented researchers towards the preparation of electro-optic thin films. These open the path to the realization of future active photonic devices like waveguide modulators with a voltage-length product lowered by orders of magnitude, or electrically tunable photonic crystals. A second motivation to prepare SBN in the form of a thin film is it makes it easier to engineer ferroelectric domains using an atomic force microscope. Domain patterns may be designed in view of optimizing a quasi-phase-matching regime for wavelength conversion.

The experimental KPFM investigations reported in this paper have been carried out on a SBN:60 film, 0.6 $\mu \mathrm{m}$ thick, (001) oriented, epitaxially grown on a $\mathrm{Pt}(001) / \mathrm{MgO}(001)$ substrate, and exhibiting two mirror symmetric in-plane orientations $\pm 18.4^{\circ}$ with respect to the $\mathrm{MgO}$ cell axes. The direction of the spontaneous polarization is perpendicular to the surface. Platinum dots were deposited on the surface, which are useful to characterize the dielectric, piezoelectric, and electro-optic properties. Figure 6 shows the experimental current intensity-voltage I(V) plots obtained using the current-sensing AFM mode (CSAFM) with the tip in contact with a platinum dot. The voltage applied between bottom electrode and tip has been increased then decreased at a constant speed $\mathrm{dV} / \mathrm{dt}$ so that the absolute value of the current is proportional to the capacitance $\mathrm{C}$ of the lever-sample system. This capacitance clearly exhibits a typical ferroelectric nonlinear $\mathrm{C}(\mathrm{V})$ behavior. Any further detail about the preparation and the characterization of SBN films can be found in the previous reports of the author. ${ }^{31-34}$

It is to be noted that SBN is a low coercive field ferroelectric material: $E_{c}$ is a few $\mathrm{kV} / \mathrm{cm}$ in $\mathrm{SBN}$ crystals, and about $6 \mathrm{kV} / \mathrm{cm}$ in the epitaxial SBN:60 film used in this work. The latter value is 20 times lower than the coercive field reported to be about $110-120 \mathrm{kV} / \mathrm{cm}$ in $\mathrm{PbZr}_{\mathrm{x}} \mathrm{Ti}_{1-\mathrm{x}} \mathrm{O}_{3}$ (PZT) epitaxial films. ${ }^{35}$ The allowed operating voltages in a PFM procedure are in the same proportion. Moreover, the

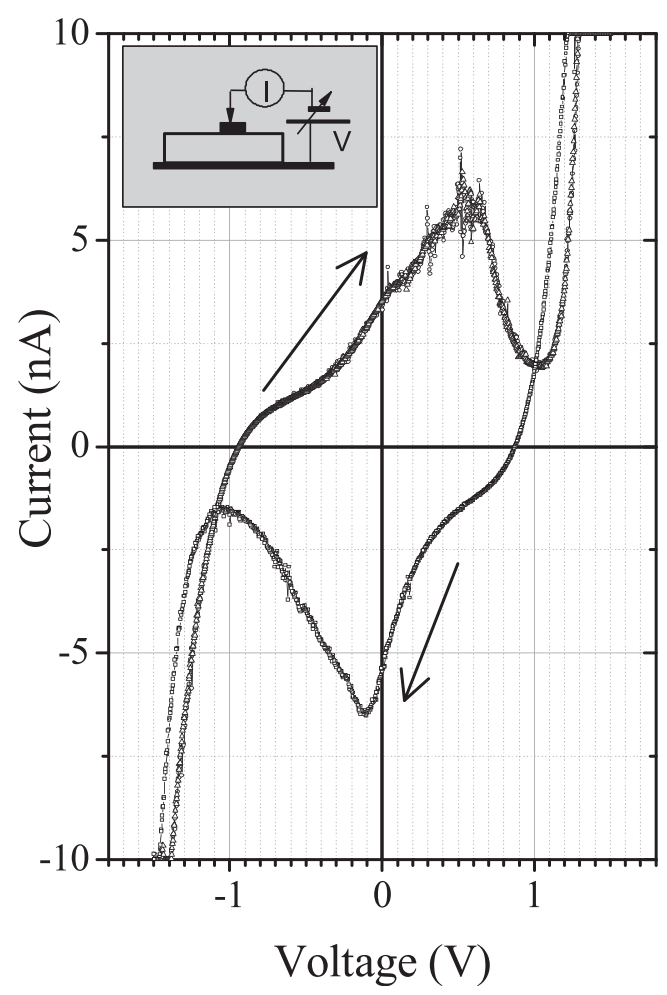

FIG. 6. Experimental current intensity-voltage I(V) plots obtained with the SBN/Pt sample using the CSAFM mode. The tip is in contact with a top platinum dot $500 \mu \mathrm{m}$ in diameter. The voltage has been increased then decreased at a constant speed $\mathrm{dV} / \mathrm{dt}$ so that the absolute value of the current is proportional to the capacitance $\mathrm{C}$ of the system. This capacitance exhibits a typical ferroelectric nonlinear $\mathrm{C}(\mathrm{V})$ behavior. 
converse piezoelectric coefficient of SBN is about 3-4 times lower than that of PZT. The amplitude of the PFM signal generated by a SBN sample is therefore expected about 60-80 times lower than that generated by a PZT sample with the same thickness. As might be therefore expected, the attempts to image a polarized area in the above micrometer thick SBN film by using the PFM technique yielded poorly contrasted images at best.

\section{B. Qualitative KPFM investigations}

The scanning probe microscope used for the experiments described in this paper is a commercial PicoPlus microscope fabricated by Molecular Imaging (now merged into Agilent Technologies), and equipped with Nanosensors $\mathrm{Pt} / \mathrm{Ir}$ coated silicon cantilevers for electrical conduction. KPFM measurements were performed in air at room temperature, using a dual-pass scheme. The tip acquires the surface topography during a first scan line using the standard intermittent contact mode feedback (amplitude modulation) at a frequency $\mathrm{f}$ near the resonance frequency $\mathrm{f}_{0}$ of the lever $\left(\mathrm{f}_{0} \approx 65 \mathrm{kHz}\right)$. The cantilever is then possibly lifted up by a distance $\mathrm{H}$ from its initial mean position $(\mathrm{H} \approx 0 \rightarrow 100 \mathrm{~nm})$, its mechanically driven oscillation is stopped, and an alternating voltage $\mathrm{V}_{\mathrm{AC}} \cos (2 \pi \mathrm{f}) \mathrm{t}$ is applied between the cantilever and the bottom electrode of the sample. The tip then scans the same line a second time at a held constant distance from the surface while the amplitude of the harmonic oscillation of the tip is continuously nullified using the Kelvin voltage feedback loop. In the experiments presented here, a drive voltage amplitude $\mathrm{V}_{\mathrm{AC}} \geq 1 \mathrm{~V}$ was required to operate with a satisfactory sensitivity. Poling is processed by applying a DC voltage $( \pm 0-10 \mathrm{~V})$ between the tip in contact with the film surface and the platinum bottom electrode.

Figure 7 shows the KPFM images of two areas of the SBN film where the three capital letters LCF have been previously "written" by moving the biased tip in contact with the surface. The topography acquired during the first pass is shown on the left of each KPFM image. The poling voltages applied to the tip were $-10 \mathrm{~V}$ (top image) and $+10 \mathrm{~V}$ (bottom image) so that the written polarization had the direction indicated below each image. The KPFM images show the variation in Kelvin voltage induced by the poling process. As expected, this variation is positive when the polarization is directed upwards and negative when it is directed downwards. If the poling process had resulted in a charge injection on the surface instead of the film poling, the contrast in the KPFM images would be the opposite of that observed. Nevertheless, the two mechanisms may have coexisted during the poling process and screening extrinsic charges may be present on the surface, either injected or simply attracted by the field above the surface. The electric field generated by the written polarization above the film may be therefore lowered by the possible presence of such extrinsic charges but it is neither cancelled nor reversed. The KPFM images of the SBN sample are always dominated by the written polarization, which is unlike what was often reported by the authors studying ferroelectric surfaces. ${ }^{10,15-20}$ A first likely reason for this difference is that the field necessary to polarize SBN

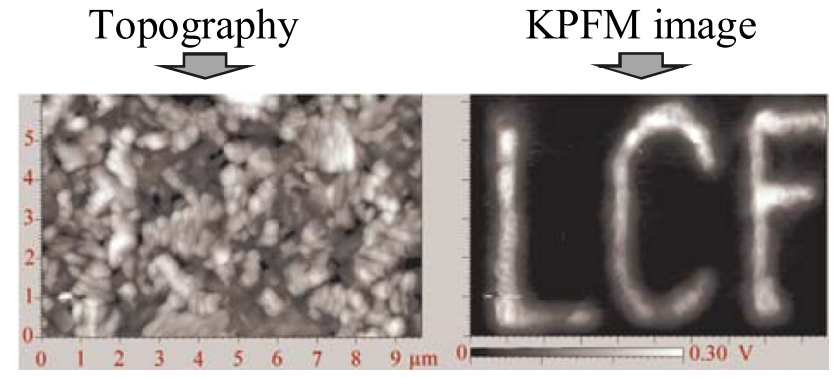

(a)

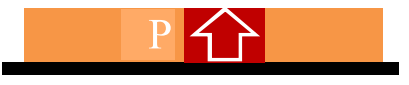

(b)

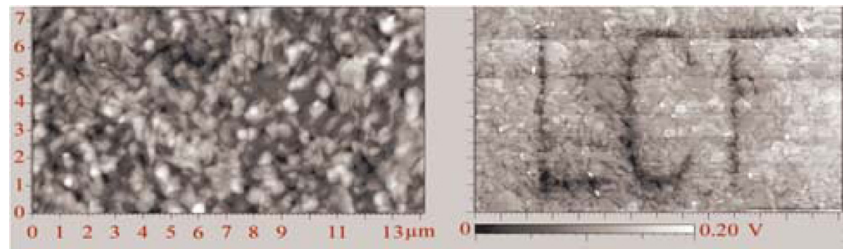

(c)

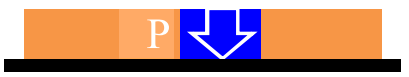

(d)

FIG. 7. KPFM images ((b) and (d)) of two areas of the SBN film where the three capital letters LCF have been previously "written" by moving the biased tip in contact with the surface. The topography acquired during the first pass is shown on the left of each KPFM image ((a) and (c)). The poling voltages applied to the tip were $-10 \mathrm{~V}$ (top image) and $+10 \mathrm{~V}$ (bottom image) so that the written polarization had the direction indicated below each image.

is about 20 times lower than that necessary to polarize materials with a high coercive field like PZT. The charge injected during the poling process of ferroelectric thin films, that was reported to screen the polarization bound charge, ${ }^{15,17,19}$ is consequently expected limited in our case. On another hand, as expected from the above calculation (see comment of Fig. 4), the screening by extrinsic charges attracted on the surface mainly affects thick ferroelectric samples. ${ }^{10,16,20}$ The possible presence of injected screening charges cannot be ruled out nor prevented by operating under vacuum. The KPFM (and EFM) measurements provide information on the resulting equivalent polarization of the film.

The KPFM contrast of a uniformly poled area is shown in Figure 8 . The mean value of the Kelvin voltage in this image is $+0.66 \mathrm{~V}$ and its RMS variation is $0.08 \mathrm{~V}$. The comparison with the topography shown on the left of the figure indicates a systematic increase of the electric field intensity straight above the grain boundaries. The same field increase is observed when the polarization has the downward direction: the grain boundaries then appear as black contours in the KPFM image. It is to be noted that a defective feedback loop would not result in a systematic excess of the $V_{K}$ absolute value whatever the variation in $\mathrm{V}_{\mathrm{K}}$ is through the grain boundary, like observed here. This electric field increase above grain boundaries suggests the existence of space charges formed near the grain surfaces during the poling process. It implies that free intrinsic charges have been moved by the poling field and trapped into localized states. Taking the grain boundaries contribution into account or not does not significantly modify the mean value of $V_{K}$ in the area. In the following quantitative 


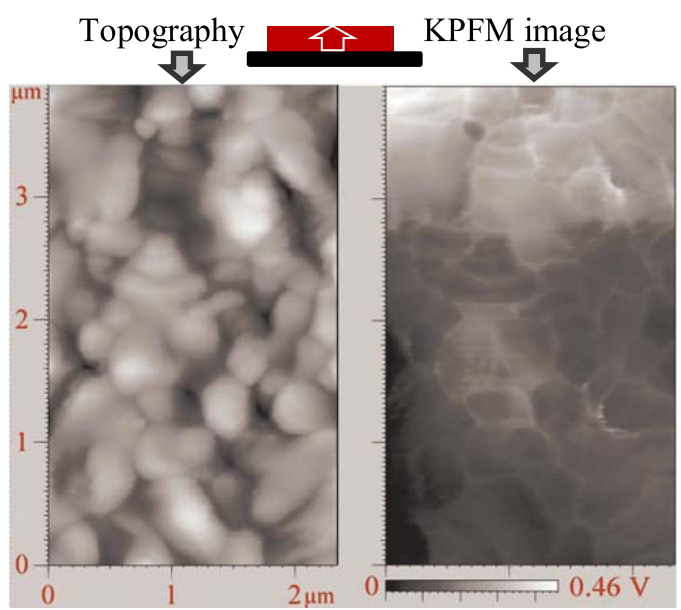

FIG. 8. Topography and KPFM image of a uniformly poled area in the SBN film. The poling procedure consisted in raster scanning the biased tip $(-10 \mathrm{~V})$ in contact with the SBN surface at a $4 \mu \mathrm{m} / \mathrm{s}$ speed. The average $\mathrm{V}_{\mathrm{K}}$ value is $+0.66 \mathrm{~V}$ and the RMS deviation is $0.08 \mathrm{~V}$. A systematic increase of the electric field above the grain boundaries may be noted.

investigations, square areas of fixed dimensions are uniformly poled and a mean Kelvin voltage is measured in a small region around the square center.

\section{Quantitative KPFM investigations}

\section{Tip-sample distance $V_{K}$ dependence}

The tip-sample distance dependence of the Kelvin voltage has been investigated by measuring $V_{K}$ above the center of square poled areas as a function of the interleave height $\mathrm{H}$. An experiment is depicted in Figure 9. Two square areas have been first uniformly poled in the SBN film using identical poling conditions. A KPFM image (Fig. 9(b)) has been then formed by continuously scanning the fixed line that connects the centers of the two squares (Fig. 9(a)). While scanning this line, the interleave height of the tip has been first increased by steps from zero to $1.2 \mu \mathrm{m}$ (bottom part of the KPFM image), then decreased back to zero using larger steps, then held constant (top part of the image). A cross section (blue arrow in the KPFM image) is shown in Fig. 9(c). It stands out from this cross section and from the top part of the KPFM image that the Kelvin voltage value at a given distance from the surface is stable during the experiment time (about $1 \mathrm{~h} 30 \mathrm{~min}$ ) and is not altered by the measurement process.

The Kelvin voltage $\mathrm{V}_{\mathrm{KNP}}$ measured above the non-poled areas of the SBN film is not zero but about $+0.12 \mathrm{~V}$ whatever the tip-surface distance is. This insensitivity to the tip-surface distance is not consistent with the hypothesis of a default polarization of the SBN film. Moreover, a polarized area of so large dimensions should generate a quasi-zero electric field. At last, the poling process should erase this hypothetical initial polarization and Kelvin voltages should be opposite when polarizations are written opposite. They are not and the assumption of a $+0.12 \mathrm{~V}$ offset well accounts for the observed asymmetry. A likely explanation is that this offset arises from the alignment of the Fermi level at the SBN/Pt interface with that of the tip and is the measurement of the induced contact potential difference. The Kelvin voltage induced by the SBN
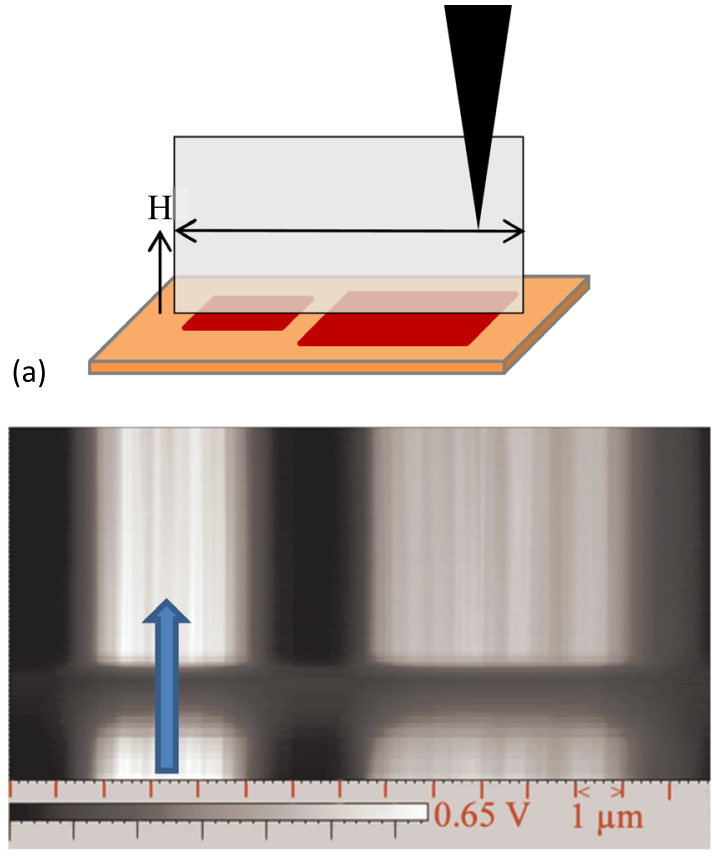

(b)

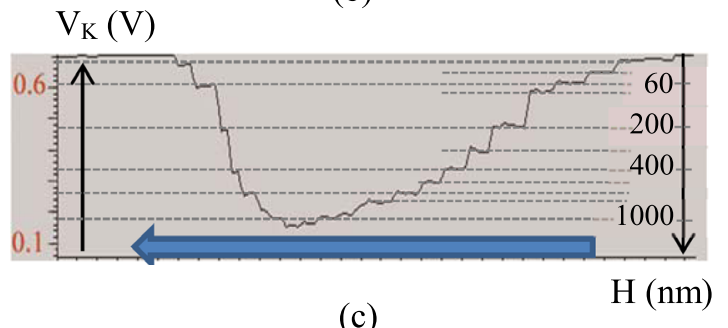

FIG. 9. KPFM signal dependence on tip-surface distance. (a) Two squares, $2.5 \mu \mathrm{m}$ and $5 \mu \mathrm{m}$ side, have been uniformly poled in the SBN film. A KPFM image has been then formed by continuously scanning a fixed line while the tip-surface distance was increased then decreased by steps. $\mathrm{H}$ is the interleave height introduced between the first (topography) and the second pass. (b) KPFM image. The top part of the image where the distance was held constant confirms that a KPFM measurement does not alter the film polarization. (c) Cross section corresponding to the blue arrow in (b).

polarization $\left(\mathrm{V}_{\mathrm{KP}}\right)$ is consequently the difference between the values measured above the poled area $\left(\mathrm{V}_{\mathrm{K}}\right)$ and above a nonpoled area $\left(\mathrm{V}_{\mathrm{KNP}}\right)$ : $\mathrm{V}_{\mathrm{KP}}=\mathrm{V}_{\mathrm{K}}-\mathrm{V}_{\mathrm{KNP}}$.

The ratio $\mathrm{V}_{\mathrm{KP}}(\mathrm{H}) / \mathrm{V}_{\mathrm{KP}}(0)$ measured above the centers of poled square areas of different sizes is plotted in Figure 10(a) as a function of $\mathrm{H}$. We experimentally verify that the Kelvin voltage induced by a given polarization in the film depends on both the tip-surface distance and the poled area size. For comparison, Figure 10(b) shows the ratio $\mathrm{E}(\mathrm{H}) / \mathrm{E}(0)$ calculated from Eq. (23), where $\mathrm{E}$ is the electric field generated by the polarized area at the position of the tip apex. The mean tip-surface distance during the acoustic first pass was fixed so that the tip- surface distance during the second pass is $\mathrm{Z}_{0}(\mu \mathrm{m})=\mathrm{H}+0.02$. It stands out from this comparison that the only decrease of $E$ at the tip apex with increasing distance cannot account for the observed distance dependence of $\mathrm{V}_{\mathrm{KP}}$. The decay of $\mathrm{V}_{\mathrm{KP}}$ is steeper which implies that the relative contribution of the volume of the tip to the KPFM response is (a) not negligible and (b) decreasing with increasing tip-surface distance. 


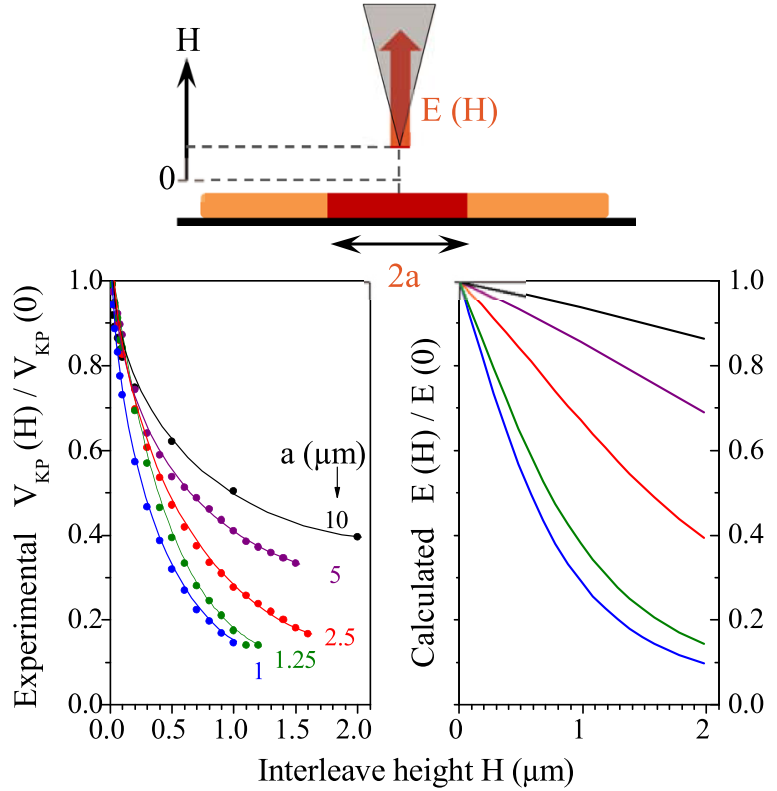

(a)

(b)

FIG. 10. (a) KPFM signal dependence on tip-surface distance measured with square poled areas of increasing size. $\mathrm{V}_{\mathrm{KP}}(\mathrm{H})$ is the KPFM voltage induced by the film polarization above the center of the poled area when the tip apex is at a distance $\mathrm{H}+0.02 \mu \mathrm{m}$ from the surface. (b) Calculated electric field $\mathrm{E}(\mathrm{H})$ at the tip apex position as a function of $\mathrm{H}$ with poled area size as a parameter. The comparison of the two layers shows that the only decay of $\mathrm{E}$ at the tip apex cannot account for the measured $\mathrm{V}_{\mathrm{KP}}$ decay.

\section{Stability of the remnant polarization $P$}

Two $5 \mu \mathrm{m} \times 5 \mu \mathrm{m}$ areas have been poled using identical poling conditions, and KPFM images have been then continuously recorded during hours. The time necessary to form one image was $1 \mathrm{~h} 20 \mathrm{~min}$. The first and the last images were of the two squares, all the other ones were of one square only. The comparison between the square that had been scanned two times only and the other one revealed no difference, which confirms that a KPFM measurement undergone during hours does not alter the film polarization. Figure 11 shows the ratio $\mathrm{V}_{\mathrm{KP}}(\mathrm{t}) / \mathrm{V}_{\mathrm{KP}}(0)=\mathrm{P}(\mathrm{t}) / \mathrm{P}(0)$ as a function of the time elapsed from poling, where $\mathrm{P}(\mathrm{t})$ is the polarization of the film at time t. The experiment has been carried out again three times with $2 \mu \mathrm{m} \times 2 \mu \mathrm{m}$ areas poled using different poling voltages $\mathrm{V}_{\mathrm{w}}$ so that the initial polarization $\mathrm{P}(0)$ was different. The results shown in Fig. 11 indicate that (a) the decay kinetics is poled area size dependent: the polarization decays faster when the polarized area is smaller; (b) the initial polarization intensity has no influence on the decay kinetics. These conclusions do agree with those inferred from PFM measurements on SBN crystals by researchers of the Russian Academy of Sciences. ${ }^{36}$ The decay kinetics measured here is relative to isolated uniformly poled areas only. The stability of, for example, a large chessboard made of contiguous reversed domains in SBN crystals has been shown ${ }^{36}$ to exceed by orders of magnitude that of an isolated square.

\section{Polarization dependence on poling parameters}

The two parameters of the poling process investigated here are the voltage $\mathrm{V}_{\mathrm{W}}$ applied between bottom electrode

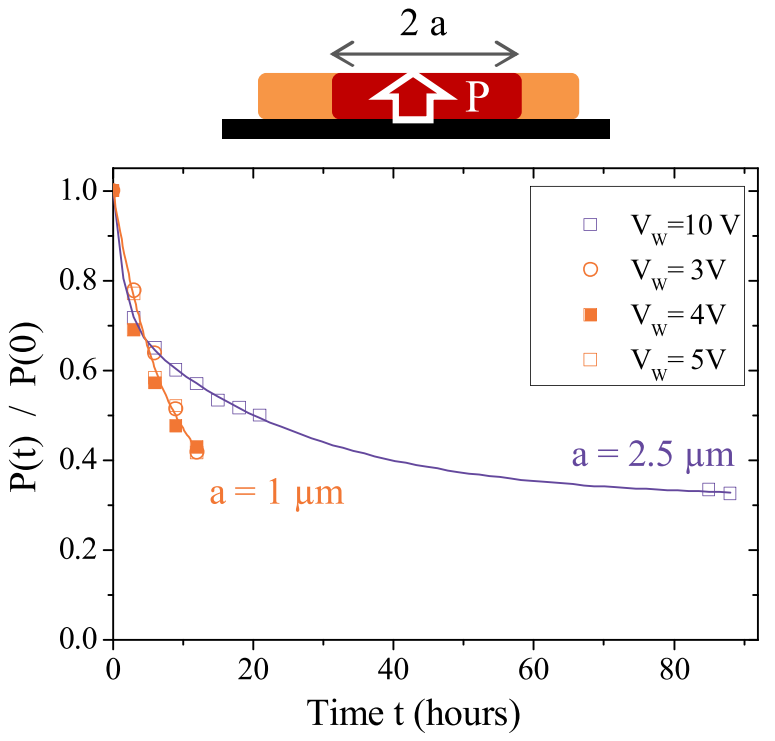

FIG. 11. Stability of the tip-induced polarization in the film. $\mathrm{V}_{\mathrm{KP}}(\mathrm{t}) / \mathrm{V}_{\mathrm{KP}}(0)$ $=\mathrm{P}(\mathrm{t}) / \mathrm{P}(0)$ has been measured above the center of square poled areas of different sizes. Given a poled area size $(2 \mu \mathrm{m} \times 2 \mu \mathrm{m})$, the initial polarization was varied by modifying the poling voltage $(3,4,5 \mathrm{~V})$. The decay kinetics appears poled area size dependent and independent of initial polarization.

and tip and the duration $\Delta \mathrm{t}_{\mathrm{W}}$ of the poling contact. Figure 12 shows the KPFM images of two areas of the SBN film where the biased tip $\left(\mathrm{V}_{\mathrm{W}}=+10 \mathrm{~V}\right)$ has been previously put in contact with the surface during $300 \mathrm{~s}$ (left column) or $450 \mathrm{~s}$ (right column). The $\mathrm{V}_{\mathrm{K}}$ scales are significantly different which proves that $300 \mathrm{~s}$ is not sufficient to obtain a point saturated polarization in the film. Although boundary effects and spatial resolution will not be discussed in the present paper, it may be noted that the Full Width at Half Maximum (FWHM) is similar in the two cross sections and about $0.70 \mu \mathrm{m}$. The origin of this width is not only in the far field generated by the film polarization but also in the very divergent electric field used to pole the film, which generates a lateral broadened polarization profile rather than a point polarization. The stable shape of the cross section allows us to infer that the $\mathrm{P}$ induced Kelvin voltage $\mathrm{V}_{\mathrm{KP}}$ straight above the contact point may be considered as proportional to $\mathrm{P}$. The experimental variation of $\mathrm{V}_{\mathrm{KP}}$ as a function of the poling time $\Delta \mathrm{t}_{\mathrm{W}}$ is shown in Figure 13. The tip poling kinetics at room temperature appears particularly slow compared to a macroscopic poling kinetics. The strongly inhomogeneous field generated by the tip in the film is responsible for this difference. The kinetics observed here is that of the vertical domain growth under the tip.

The polarization dependence on poling voltage has been investigated by successively performing the poling and imaging of square areas of identical size using identical conditions except $\mathrm{V}_{\mathrm{W}}$ so that the factor $\mathrm{K}$ in Eq. (24) was a constant. Time $(\Delta \mathrm{t})$ elapsed between poling and imaging was held constant so that $\mathrm{P}(\Delta \mathrm{t}) / \mathrm{P}(0)$ was also a constant. Well then the $\mathrm{P}$ induced Kelvin voltage measured above the center of each poled area is proportional to the initial remnant polarization $\mathrm{P}(0)$ in this area. Figures $14(\mathrm{a})$ and $14(\mathrm{~b})$ show the results obtained with two series of $5 \times 5 \mu \mathrm{m}^{2}$ and $2 \times 2 \mu \mathrm{m}^{2}$ poled areas, respectively. The maximum mean 
Topography
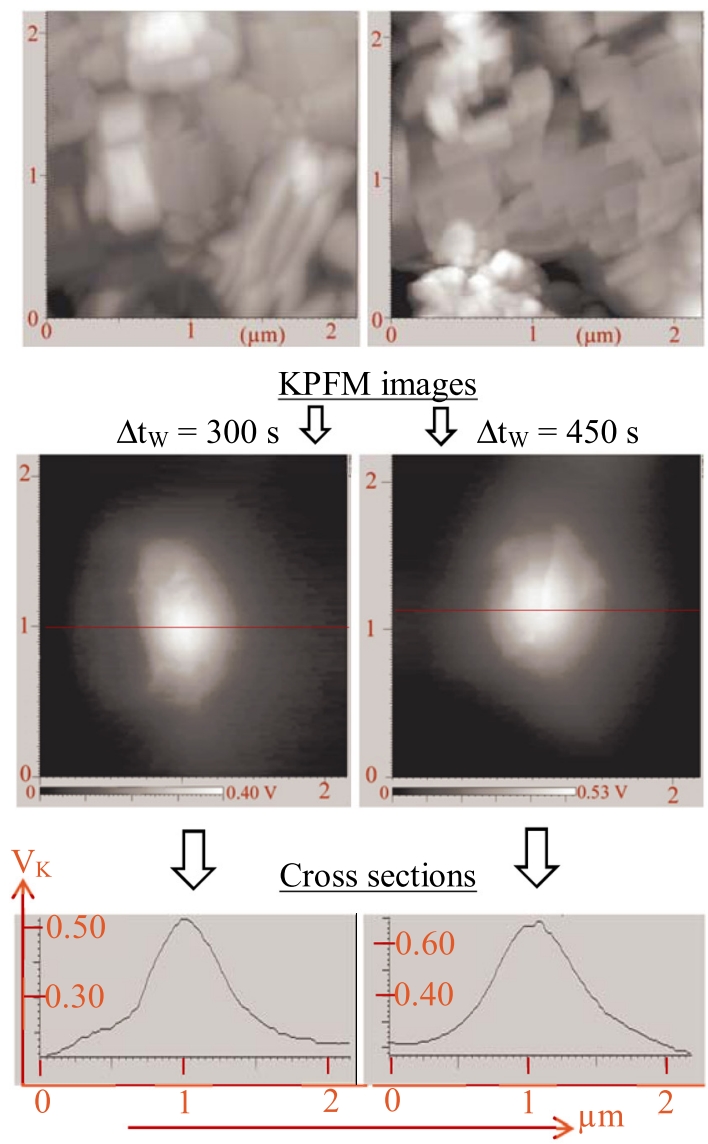

FIG. 12. Influence of the poling contact duration. The biased tip $(-10 \mathrm{~V}$ applied to the tip) was put in contact with the SBN surface during $300 \mathrm{~s}$ (left) or $450 \mathrm{~s}$ (right). The two KPFM images centered on the contact points indicate that the added poling duration has still significantly increased the polarization in the film.

poling field $\left(E_{m}=V_{W} / d\right)$ applied to the $S B N$ film in these experiments appears to be not sufficient to saturate the polarization.

For comparison, the macroscopic remnant polarization in the SBN film has been measured versus poling field from the hysteresis cycles drawn using a standard Sawyer-Tower circuit and a Pt dot $500 \mu \mathrm{m}$ in diameter as top contact. The poling

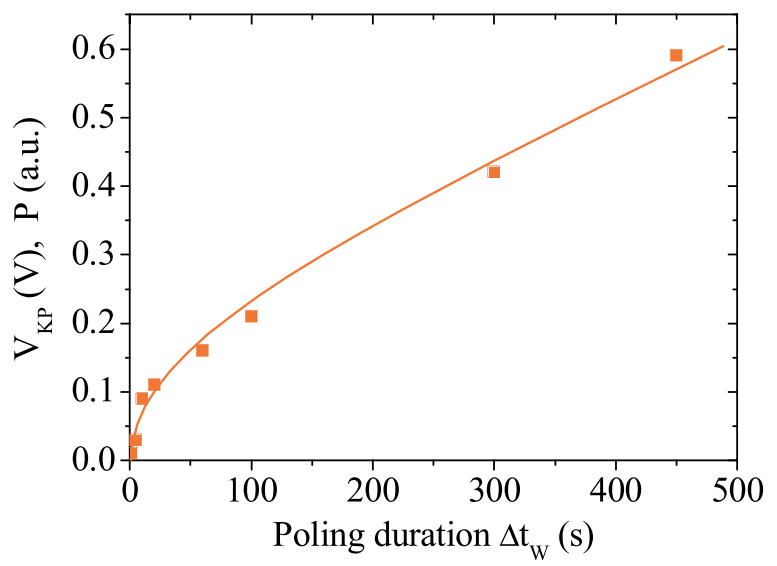

FIG. 13. Tip-induced polarization as a function of the poling contact duration. Experiments are described in Fig. 12.

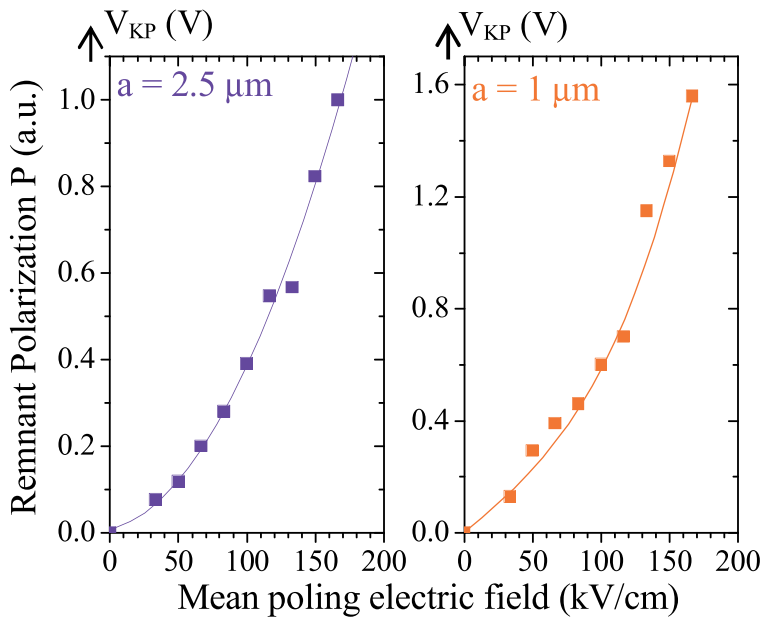

(a)

(b)

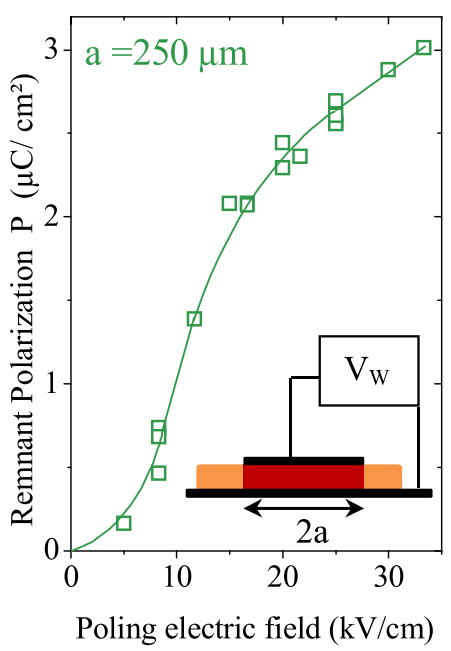

(c)

FIG. 14. Influence of the poling voltage. Square areas of identical size have been uniformly poled then imaged using identical conditions except the poling voltage $\mathrm{V}_{\mathrm{W}}$ so that the KPFM signal $\mathrm{V}_{\mathrm{KP}}$ was proportional to the polarization P. (a) $\mathrm{V}_{\mathrm{KP}}$ measured above the center of $5 \mu \mathrm{m} \times 5 \mu \mathrm{m}$ or (b) $2 \mu \mathrm{m} \times 2 \mu \mathrm{m}$ poled areas as a function of the mean poling field $\left(\mathrm{V}_{\mathrm{W}} / \mathrm{d}\right)$. (c) Macroscopic remnant polarization in the SBN film versus poling field measured from hysteresis cycles drawn using a standard Sawyer-Tower circuit and a Pt dot $500 \mu \mathrm{m}$ in diameter as top contact.

voltage $\mathrm{V}_{\mathrm{W}}$ was then the amplitude of the drive voltage sweep. Figure 14(c) displays the value of this macroscopic remnant polarization versus the mean poling field in the film. It stands out from the comparison between microscopic and macroscopic measurements that the mean field necessary to saturate the film polarization is significantly lower in the macroscopic configuration. The plots over the range of $0-170 \mathrm{kV} / \mathrm{cm}$ in Figures 14(a) and 14(b) could be fitted to the plot over the range of $0-10 \mathrm{kV} / \mathrm{cm}$ in Figure 14(c). The explanation for this considerable difference is again to be found in the strongly inhomogeneous field generated by the poling tip. If we assume that this field varies in the film depth approximately like that generated by a point charge on the surface, the field near the bottom electrode $\left(0.6 \mu \mathrm{m}\right.$ from the tip apex) is about $10^{2}$ times lower than the mean field in the film. This implies that the vertical size of the domain generated under the tip increases with 
the mean poling field, and should reach the film thickness only if this mean poling field is about $10^{2}$ times larger than the coercive field, that is more than $600 \mathrm{kV} / \mathrm{cm}\left(\mathrm{E}_{\mathrm{c}} \approx 6 \mathrm{kV} / \mathrm{cm}\right.$ in the SBN:60 films). No sign of saturation should be expected with a lower mean poling field. Well then we do not observe such a sign with fields as high as $170 \mathrm{kV} / \mathrm{cm}$ (Figs. 14(a) and 14(b)). On the contrary, the field is uniform in the standard macroscopic measurements and the coercive field is applied to the whole film as soon as the mean poling field reaches the coercive value.

On another hand, the depolarizing field is reduced by the top electrode screening charges in the macroscopic measurements while it is not in KPFM measurements. The remnant polarization produced by a given uniform poling field is consequently expected larger in the macroscopic configuration. Since the factor K in Eq. (24) is not calculable, only a calibration procedure would enable to infer an absolute value of the polarization from a $V_{K}$ measurement. The preceding considerations point out the difficulty implementing any calibration procedure. They also might explain the difference usually observed in the literature between the macroscopic and the PFM values of the converse piezoelectric coefficient.

It is to be noted that in our KPFM experiments the polarization was therefore not uniform in the depth of the film so that the polarization plotted in Figures 11, 13, 14(a), and 14(b) is the uniform polarization that would produce the same electric field above the surface as the actual polarization profile in the film.

\section{CONCLUSION}

The ability of non-contact electric modes (KPFM and EFM) to provide quantitative information about the remnant polarization in a ferroelectric thin film has been theoretically and experimentally investigated. The theoretical relationship established between the microscopic signal and the polarization in the film is linear in the KPFM mode and parabolic in the EFM mode. The polarization dependence on any variable relative to the film preparation conditions or to the poling process can be quantitatively investigated using KPFM provided the tip-shape, tip-surface distance, and poled area dimensions are held constant. On the contrary, KPFM and EFM cannot provide neither an absolute value of the polarization nor direct information about the polarization dependence on poled area geometry.

Based on these conclusions, experimental KPFM investigations have been carried out on a ferroelectric strontium barium niobate $\left(\mathrm{Sr}_{\mathrm{x}} \mathrm{Ba}_{1-\mathrm{x}} \mathrm{Nb}_{2} \mathrm{O}_{6}, \mathrm{SBN}: 100 \mathrm{x}\right)$ epitaxial thin film. The dependence of the polarization in the film on poling duration, poling voltage, and time elapsed from poling has been quantitatively investigated, with the poled area dimensions as a parameter. For comparison, the macroscopic remnant polarization in the SBN film has been measured versus poling field from hysteresis cycles drawn using a macroscopic Pt dot as top contact. The significant difference observed between macroscopic and microscopic results can be accounted for by using a simple model of the inhomogeneous electric field generated by a poling tip.
KPFM measurements have been tested in this work not to alter the initial polarization of the film even when carried out during hours, and to be able to provide essential qualitative and quantitative information on the equivalent uniform polarization in a ferroelectric thin film.

${ }^{1}$ M. Nonnenmacher, M. P. O'Boyle, and H. K. Wickramasinghe, Appl. Phys. Lett. 58(25), 2921-2923 (1991).

${ }^{2}$ W. Melitz, J. Shen, A. C. Kummel, and S. Lee, Surf. Sci. Rep. 66(1), 1-27 (2011).

${ }^{3}$ L. Kelvin, Philos. Mag. 46, 82 (1898).

${ }^{4}$ W. A. Zisman, Rev. Sci. Instrum. 3, 367 (1932).

${ }^{5}$ J. Colchero, A. Gil, and A. M. Baro, Phys. Rev. B 64, 245403 (2001).

${ }^{6}$ T. R. Albrecht, P. Grütter, D. Horne, and D. Rugar, J. Appl. Phys. 69(2), 668 (1991).

${ }^{7}$ S. Hudlet, M. Saint Jean, B. Roulet, J. Berger, and C. Guthmann, J. Appl. Phys. 77(7), 3308 (1995).

${ }^{8}$ Y. Rosenwaks, R. Shickler, T. Glatzel, and S. Sadewasser, Phys. Rev. B 70, 085320 (2004).

${ }^{9}$ L. N. Kantorovich, A. I. Livshits, and M. Stoneham, J. Phys.: Condens. Matter 12, 795 (2000).

${ }^{10}$ S. V. Kalinin and D. A. Bonnell, Phys. Rev. B 63, 125411 (2001).

${ }^{11}$ F. Bocquet, L. Nony, C. Loppacher, and T. Glatzel, Phys. Rev. B 78, 035410 (2008).

${ }^{12}$ A. Masago, M. Tsukada, and M. Shimizu, Phys. Rev. B 82, 195433 (2010).

${ }^{13}$ R. Baier, C. Leendertz, M. Ch. Lux-Steiner, and S. Sadewasser, Phys. Rev. B 85, 165436 (2012).

${ }^{14}$ A. Sadeghi, A. Baratoff, S. A. Ghasemi, S. Goedecker, T. Glatzel, S. Kawai, and E. Meyer, Phys. Rev. B 86, 075407 (2012).

${ }^{15}$ X. Q. Chen, H. Yamada, T. Horiuchi, K. Matsushige, S. Watanabe, M. Kawai, and P. S. Weiss, J. Vac. Sci. Technol. B 17(5), 1930 (1999).

${ }^{16}$ S. V. Kalinin and D. A. Bonnell, Appl. Phys. Lett. 78(8), 1116 (2001).

${ }^{17}$ J. Y. Son, S. H. Bang, and J. H. Cho, Appl. Phys. Lett. 82(20), 3505 (2003).

${ }^{18}$ R. Shao, M. P. Nikiforov, and D. A. Bonnell, Appl. Phys. Lett. 89, 112904 (2006).

${ }^{19}$ H. Guo, G. Cheng, S. Wang, S. Dai, S. Wu, S. Zhou, Y. Li, and Z. Du, Appl. Phys. Lett. 97, 162902 (2010).

${ }^{20}$ D. Y. He, L. J. Qiao, A. A. Volinsky, Y. Bai, and L. Q. Guo, Phys. Rev. B 84, 024101 (2011).

${ }^{21}$ F. Yan, G. Z. Xing, M. Islam, S. Li, and L. Lu, Appl. Phys. Lett. 100, 172901 (2012).

${ }^{22}$ H. Choi, J. Hong, and K. No, Appl. Phys. Lett. 101, 042904 (2012).

${ }^{23}$ P. Güthner and K. Dransfeld, Appl. Phys. Lett. 61(9), 1137 (1992).

${ }^{24}$ S. V. Kalinin and D. A. Bonnell, Phys. Rev. B 65, 125408 (2002).

${ }^{25}$ A. N. Morozovska, E. A. Eliseev, S. L. Bravina, and S. V. Kalinin, J. Appl. Phys. 110, 052011 (2011).

${ }^{26}$ J. E. Stern, B. D. Terris, H. J. Mamin, and D. Rugar, Appl. Phys. Lett. 53(26), 2717 (1988).

${ }^{27}$ H. Bluhm, A. Wadas, R. Wiesendanger, K. P. Meyer, and L. Szczesniak, Phys. Rev. B 55(1), 4 (1997).

${ }^{28}$ Y. Martin, C. C. Williams, and H. K. Wickramasinghe, J. Appl. Phys. 61(10), 4723 (1987).

${ }^{29}$ P. B. Jamieson, S. C. Abrahams, and J. L. Bernstein, J. Chem. Phys. 48(11), 5048 (1968).

${ }^{30}$ P. V. Lenzo, E. G. Spencer, and A. A. Ballman, Appl. Phys. Lett. 11(1), 23 (1967).

${ }^{31}$ M. Cuniot-Ponsard, J. M. Desvignes, B. Ea-Kim, and E. Leroy, J. Appl. Phys. 93(3), 1718 (2003).

${ }^{32}$ M. Cuniot-Ponsard, J. M. Desvignes, and A. Bellemain, J. Mater. Sci. 41, 5302 (2006).

${ }^{33}$ M. Cuniot-Ponsard, J. M. Desvignes, and A. Bellemain, J. Appl. Phys. 109, 014107 (2011).

${ }^{34}$ M. Cuniot-Ponsard, Ferroelectrics-Material Aspects (InTech, see http:// www.intechopen.com, 2011), Chap. 23.

${ }^{35}$ S. Bühlmann, E. Colla, and P. Muralt, Phys. Rev. B 72, 214120 (2005).

${ }^{36}$ T. R. Volk, L. V. Simagina, R. V. Gainutdinov, A. L. Tolstikhina, and L. I. Ivleva, J. Appl. Phys. 108, 042010 (2010). 